\title{
Vector saliva in vaccines for visceral leishmaniasis: a brief encounter of high consequence?
}

\section{Shaden Kamhawi *, Hamide Aslan and Jesus G. Valenzuela*}

Vector Molecular Biology Section, Laboratory of Malaria and Vector Research, National Institute of Allergy and Infectious Diseases, National Institutes of Health, Rockville, MD, USA

\section{Edited by:}

Nahid Ali, Indian Institute of Chemical

Biology, India

Reviewed by:

Mauricio Martins Rodrigues, Federal University of São Paulo, Brazil

Alexandre Barbosa Reis, Universidade Federal de Ouro Preto, Brazil

\section{*Correspondence:}

Shaden Kamhawi and Jesus G.

Valenzuela, Vector Molecular Biology Section, Laboratory of Malaria and

Vector Research, National Institute of Allergy and Infectious Diseases,

National Institutes of Health, 12735

Twinbrook Parkway, Room 2E22,

Rockville, MD 20878, USA

e-mail:skamhawi@niaid.nih.gov;

jvalenzuela@niaid.nih.gov
Visceral leishmaniasis $(V L)$ is a vector-borne disease transmitted by phlebotomine sand flies and remains the most serious form of the disease with no available human vaccine. Repeatedly, studies have demonstrated the immunogenicity and protective efficacy of a number of sand fly salivary proteins against cutaneous and visceral leishmaniasis. All Leishmania species including agents of $\mathrm{VL}$ are co-deposited into the skin together with vector saliva. Generally, the immune response to a protective salivary protein in vaccinated animals is rapid and possibly acts on the parasites soon after delivery into the skin by the bite of an infective sand fly. This is followed by the development of a stronger Leishmania-specific immunity in saliva-vaccinated animals compared to controls. Considering that several of the most efficacious protective molecules were identified from a proven vector of $\mathrm{VL}$, we put forward the notion that a combination vaccine that includes a Leishmania antigen and a vector salivary protein has the potential to improve vaccine efficacy by targeting the parasite at it most vulnerable stage just after transmission.

Keywords: visceral leishmaniasis, sand fly vectors, vector-transmission, salivary proteins as vaccines, Th1 immune response, delayed-type hypersensitivity response

\section{BACKGROUND}

Visceral leishmaniasis (VL), also known as kala-azar, is a systemic vector-borne neglected disease that is fatal if left untreated. There are an estimated 300,000 cases of VL globally with over 20,000 deaths per year, a statistic second only to malaria among parasitic diseases (1). Over $90 \%$ of VL cases occur in six countries (Bangladesh, Brazil, Ethiopia, India, South Sudan, and Sudan) where about 300 million people are at risk of infection $(1,2)$. From 2009 to 2012, an epidemic in South Sudan caused over 28,300 cases and nearly 900 deaths $^{1}$. Other areas have also been affected by recent persistent epidemics of VL in Ethiopia and Kenya ${ }^{1}$.

Visceral leishmaniasis is caused either by Leishmania donovani or $L$. infantum. VL caused by $L$. donovani is prevalent in East Africa and the Indian sub-continent and is considered an anthroponosis, while VL caused by L. infantum is prevalent in South Europe, North Africa, parts of the Middle East and Latin America (3-6). Phlebotomine sand flies are still considered the primary and stable mode of VL transmission. Different species of sand flies have been incriminated as vectors of VL. Phlebotomus argentipes is the only known vector of $L$. donovani in the Indian sub-continent (7-9) and P. orientalis represents the main sand fly species transmitting $L$. donovani within countries of East Africa, Saudi Arabia, and Yemen $(10,11)$. On the other hand, there are several proven vectors of VL in the Eastern Mediterranean among which $P$. ariasi and $P$. perniciosus represent the primary species transmitting $L$. infantum $(12,13)$, while Lutzomyia longipalpis is considered the primary vector of L. infantum across Latin America $(14,15)$.

${ }^{1}$ www.who.int/leishmaniasis
Despite, its wide distribution and high mortality rate, there are no available human vaccines against VL. Even with recent improvement in treatment (16-19) and the gates initiative for the elimination of VL from the Indian sub-continent ${ }^{2}$, there remains a need to develop a vaccine, particularly when considering the prevalence of infected individuals with subclinical infections that potentially present an uncontrolled source of parasites for the sand fly vector (20). Though the primary function of vector saliva is to facilitate blood feeding (21), a good body of evidence has shown that it modulates host immunity altering the outcome of infection with Leishmania and under certain circumstances, protecting from disease (22-24). Here, we give our perspective on the relevance of vector saliva in the transmission of and for vaccines against $\mathrm{VL}$.

\section{VECTOR SALIVA AND PROTECTION FROM LEISHMANIASIS}

Vaccination with certain immunogenic proteins in saliva of vector sand flies confers protection from leishmaniasis (25-35). Protective molecules have mostly shared a similar property, the induction of a delayed-type hypersensitivity (DTH) response biased toward a Th1 profile. Importantly, animals previously exposed to saliva or vaccinated with a Th1-biased DTH-inducing salivary protein were protected against challenge by infected vector bites $(28,30$, 35). This is significant since Peters et al. (36) showed that the innate immune response following sand fly transmission varied significantly from the response induced by needle challenge primarily related to a persistence of a neutrophilic infiltrate at the site of bite enhancing parasite virulence. Additionally, the enhanced virulence of vector-transmission was shown to abrogate protection

${ }^{2}$ www.gatesfoundation.org 
by Leishmania vaccines tested against needle challenge with parasites largely due to the need for a rapid effector immune response (37). Thus, saliva-mediated protection from vector-transmitted leishmaniasis suggests that the immune response to salivary proteins is rapid enough to restrict the establishment of Leishmania parasites following vector-challenge. Furthermore, the protection against vector-challenge displayed by animals vaccinated with a defined recombinant salivary protein indicates that the native protein despite its presence among others in saliva of the vector initiated an efficient recall response upon its co-deposition in skin with the parasites (28).

Recently, a study investigating the value of combining a protective salivary vaccine with promising Leishmania antigens tested several combinations of PpSP15, a protective salivary protein from $P$. papatasi $(31,33)$, with live recombinant $L$. tarentolae stably expressing the cysteine proteinases CPA and CPB (38). In both $\mathrm{BALB} / \mathrm{c}$ and $\mathrm{C} 57 \mathrm{BL} / 6$ mice, the animals primed with PpSP15 DNA and boosted with PpSP15 DNA and live recombinant CPA/CPB-expressing $L$. tarentolae exhibited the strongest protection against $L$. major infection followed by the group immunized with both PpSP15 and CPA/CPB-expressing L. tarentolae injected in independent sites (38). This study is the first to demonstrate the enhanced protection from leishmaniasis resulting from the inclusion of a vector salivary component to the vaccine.

The significance of vector salivary proteins in Leishmania vaccines is made more credible by the observed immunogenicity of saliva in exposed humans (39-41). Gomes et al. (39) first reported on the association between the appearance of antibodies to L. longipalpis saliva and the development of a protective cell-mediated immunity to L. chagasi. In another study, volunteers experimentally exposed to L. longipalpis produced distinct skin reactions at the bite site and displayed an increased frequency of IFN- $\gamma$ - and IL-10-producing T cells (40). Additionally, the authors demonstrated that PBMC from volunteers maintained an efficient recall response 1 year after their first exposure and produced IFN- $\gamma$ upon in vitro stimulation with saliva that was associated to a significant reduction in macrophage infection rates with $L$. chagasi. More recently, we demonstrated that the DTH response in individuals naturally exposed to bites of $P$. duboscqi, another vector sand fly, persists to mid life (41). Importantly, though PBMC from volunteers showed a Th1, Th2, or a mixed response upon in vitro stimulation with saliva, dermal biopsies from bite sites with a DTH response were dominated by macrophages and lymphocytes and exhibited an abundance of IFN- $\gamma$ indicative of a Th1 milieu (41). Though more studies in humans are needed, the above results demonstrate that repeated exposure to sand fly saliva alters the immune response of humans to the parasites co-deposited into the wound at the site of an infected bite.

\section{TRANSMISSION OF VISCERAL LEISHMANIASIS AND VECTOR SALIVA}

Despite reports of vertical transmission of L. infantum (42), it is still accepted that VL, caused by L. donovani or L. infantum, is mostly transmitted by bite of infected phlebotomine sand flies. At the site of bite, the sand fly deposits few parasites (43-45) alongside saliva in the skin. Therefore, though pathology of VL is ultimately the result of failure of internal organs, mainly the spleen and liver, there is a vital phase early after transmission where the few parasites deposited in the skin are at their most vulnerable. We believe it is at this stage that immunity to a salivary protein can potentially exert a profound effect on the survival and ability of the parasites to visceralize. Studies have identified immunogenic salivary proteins from important VL vectors that induce a distinct Th1DTH response predictive of protection from leishmaniasis (27, $29,31,46)$. In the only study investigating the potential of salivary proteins to protect against VL, LJM19, a Th1-DTH-inducing salivary protein from $L$. longipalpis, a VL vector, conferred powerful protection against progressive VL in vaccinated hamsters (29). LJM19-vaccinated animals displayed a high IFN- $\gamma /$ TGF- $\beta$ ratio and inducible NOS expression in the spleen and liver associated to a controlled parasite burden and survival up to 5 months post-infection. In contrast, controls and hamsters vaccinated with other salivary molecules developed progressive fatal VL within the same time frame (29). The long-term systemic protection from L. chagasi ( L. infantum) conferred by immunity to LJM19 was likely driven by the initial immune response to LJM19 in the skin where a distinct DTH response with high expression of IFN- $\gamma$ was observed $48 \mathrm{~h}$ after challenge with uninfected sand flies (29). Due to a shorter course of infection and the ease of assessing disease burden most studies of the protective capacity of immunogenic salivary proteins from saliva of L. longipalpis were tested using CL infection models producing promising results. Mice vaccinated with maxadilan, the vasodilator from L. longipalpis saliva protected mice against L. major infection (34), while vaccination with LJM19, protected hamsters against infections with $L$. braziliensis co-injected with saliva of the natural vector $L$. intermedia (32). LJM11, another Th1-DTH-inducing salivary protein from $L$. longipalpis, conferred partial protection against L. infantum in hamsters (29) and a strong protection against infections initiated by needle or vector-challenge with $L$. major in mice (28, 47). Table 1 provides a summary of potential salivary vaccines identified from VL vectors to date.

Studies carried out using CL models of infection have demonstrated that the initial immune response directed against sand fly saliva or one of its proteins gives rise to an accelerated and potent immune response specific to the Leishmania parasite $(28,31)$. The initial saliva-specific immune response is observed as early as 2$6 \mathrm{~h}$ after bite up to 1 week post-challenge (29-31, 35). By 2-weeks post-infection, animals vaccinated with a salivary protein mount a stronger Leishmania-specific immunity with minimized pathology $(28,31)$. This supports our hypothesis that the initial immune response to a salivary protein in the skin can potentially alter the nature of the immune response to the parasites long-term and is therefore relevant for protection against both CL and VL.

\section{VECTOR SALIVA IN A VACCINE FOR VISCERAL LEISHMANIASIS \\ RATIONALE}

From the above, immunity to a vector salivary protein can potentially have an enormous impact on progression of VL. Visceralizing parasites are initially inoculated into the skin then navigate their way to the viscera in a poorly understood manner. Assuming that 
Table 1 | Vaccine candidates identified from saliva of visceral leishmaniasis vectors.

\begin{tabular}{|c|c|c|c|c|c|}
\hline Sand fly species & Salivary molecule & Immunogenicity & Protection & Animal model & Reference \\
\hline L. longipalpis & LJM19 & Th1/DTH & L. infantum, L. braziliensis & Hamster & $(29,32)$ \\
\hline L. longipalpis & LJM11 & Th1/DTH, lgG2a & L. major & Mouse & $(28,47)$ \\
\hline L. longipalpis & LJM17 & Th1/DTH, lgG2a & L. infantum & Dog & $(27)$ \\
\hline P. ariasi & ParSP03 & DTH, IgG2a & & Mouse & (46) \\
\hline P. ariasi & ParSP25 & DTH, IgG1 & & Mouse & (46) \\
\hline
\end{tabular}

for a brief period of time these parasites are in the skin, low in number, and in close proximity to co-inoculated salivary proteins, a vaccine strategy involving immunization with a Th1-inducing salivary protein that would initiate a rapid immune response to itself at the site of bite will adversely impact the vulnerable Leishmania parasites while still in the skin. Such a vaccine could potentially enhance the efficacy of a VL vaccine by introducing an additional stage in which the parasites are attacked.

\section{DIVERSITY OF VL FOCI}

The complexity of VL transmission would clearly have an impact on the design and practicality of a salivary vaccine. L. donovani, considered an anthroponosis, is transmitted by only one species of sand flies in the Indian sub-continent but has multiple vectors in East Africa $(7-11,48)$. A similar situation exists for zoonotic VL due to L. infantum where across Latin America transmission is mostly by a single primary vector while along the Eastern Mediterranean, over six species of sand flies have been incriminated as major VL vectors (12-15). Foci where transmission involves multiple vectors would be more challenging compared to those where a vaccine needs to target a single vector species. Under these conditions, the future for salivary antigens is most likely in vaccines tailored for specific regions. Nonetheless, in several of the most important foci of VL including India, Sudan, and Latin America there is but one primary vector sand fly species, $P$. argentipes, P. orientalis, and L. longipalpis, respectively (8-10, 14, 15, 49), a situation where a tailored vaccine may be justified.

\section{CHALLENGES AND SOLUTIONS}

As mentioned above, in foci with a primary vector, inclusion of a salivary protein in a leishmaniasis vaccine can potentially enhance its efficacy. On the other hand, certain VL foci such as those in the Eastern Mediterranean region have multiple incriminated VL vectors $(12,48)$. For such foci, a salivary molecule with the appropriate immunogenicity needs to have close homologs in most sympatric vector species, creating a considerable obstacle. We are now addressing whether priming with a salivary protein and boosting with both the salivary antigen and a Leishmania antigen will drive a Leishmania-specific immunity strong enough to overcome the obstacle presented by specificity of vector salivary molecules. If successful, incorporating the best of the immunogenic salivary proteins with the most promising Leishmania antigens may present an opportunity for a pan leishmaniasis vaccine. Here, we must underscore that though a robust immunity to Leishmania driven by a preceding immunity to saliva has been demonstrated $(28,31)$, it was always generated by a challenge with virulent live parasites. It remains to be validated whether a similar level of protective immunity can be achieved with a single antigen. Considering the payback, it is a question worthy of further exploration.

\section{FURTHER CONSIDERATIONS}

Identifying salivary molecules from VL vectors that can induce a Th1-biased immunity in humans should be prioritized. Expression libraries of the secreted salivary proteins of several VL vectors are available $(46,50-53)$ and high throughput expression of endotoxin-free recombinant proteins of high purity has been achieved $(28,54)$. Developing a rapid screening assay using PBMC of healthy exposed volunteers stimulated with recombinant salivary proteins from VL vectors could rapidly reveal immunogenic antigens appropriate for further exploration as protective vaccine candidates using animal models. Additionally, we recently developed a hamster model of vector-transmitted progressive VL (55) that can further facilitate the prioritization of salivary vaccine candidates found immunogenic in humans. Here, it is important to emphasize the need to begin the search for a vaccine candidate using human cells (56). Multiple leishmaniasis vaccine candidates protected various animal models but failed to protect humans (57). This is not surprising considering that the initiation of a Th1 cellular immunity such as that induced by salivary molecules and required for protection against leishmaniasis implies efficient recognition of specific antigenic epitopes by human leukocyte antigen I (HLA-I) and HLA-II molecules for presentation to T cells (58). However, unlike anthroponotic VL where humans are the only vaccine target, zoonotic VL needs to target dogs as the domestic reservoirs and the primary source of infection to sand flies and humans $(12,48,59,60)$. Therefore, in addition to humans, salivary molecules immunogenic in dogs such as those reported for L. longipalpis (27), should also be considered for a canine vaccine.

\section{SHADES OF GRAY}

Though, we tend to put Leishmania species in clear-cut categories, nature tells us otherwise. The unique polymorphic nature 
of leishmaniasis and the plasticity of Leishmania parasites continue to confound efforts toward disease control. There are several reports where a single parasite strain commonly causing dermatotropic symptoms manifests as a visceral infection and vice versa (61-63). Specifically, we still do not understand why L. infantum, associated mainly with VL, causes only cutaneous disease in some regions (64). Similarly, L. donovani zymodeme MON-37, the parasite strain previously associated exclusively with VL in India and East Africa, has been identified as the causative agent in recently established foci of CL in Sri Lanka $(65,66)$. These unusual manifestations of leishmaniasis clearly demonstrate how little we understand the factors contributing to disease. The fact that dermotropic L. infantum genotypes can disseminate and cause severe VL in immunosuppressed individuals is indicative of the importance of host susceptibility in the outcome of infection with Leishmania parasites (67). But is the etiology of leishmaniasis mainly due to host immunity or are environmental pressures, vector-derived factors and evolution of the parasite itself equally significant? Most likely the form of leishmaniasis contracted is the consequence of all the aforementioned factors. Hence, we need to keep an open mind in our search for vaccines and perhaps entertain the option of a tailored vaccine enhanced by a salivary component of a primary vector in foci of high morbidity and mortality.

\section{CONCLUSION}

To date, a human vaccine against any form of leishmaniasis is nonexistent. There is strong evidence that certain proteins in sand fly vector saliva can: (1) induce a Th1-DTH immune response; (2) protect against both CL and VL; (3) protect against vectorinitiated CL; and (4) induce a Leishmania-specific robust immunity after challenge with minimized pathology. Considering the above, should not salivary proteins of vector sand flies be given serious consideration as candidate components in a Leishmania vaccine?

\section{ACKNOWLEDGMENTS}

This work was supported by the Intramural Research Program at the National Institute of Allergy and Infectious Diseases, National Institutes of Health.

\section{REFERENCES}

1. Chappuis F, Sundar S, Hailu A, Ghalib H, Rijal S, Peeling RW, et al. Visceral leishmaniasis: what are the needs for diagnosis, treatment and control? Nat Rev Microbiol (2007) 5(11):873-82. doi:10.1038/nrmicro1748

2. Alvar J, Velez ID, Bern C, Herrero M, Desjeux P, Cano J, et al. Leishmaniasis worldwide and global estimates of its incidence. PLoS One (2012) 7(5):e35671. doi:10.1371/journal.pone.0035671

3. Lukes J, Mauricio IL, Schonian G, Dujardin JC, Soteriadou K, Dedet JP, et al. Evolutionary and geographical history of the Leishmania donovani complex with a revision of current taxonomy. Proc Natl Acad Sci U S A (2007) 104(22):9375-80. doi:10.1073/pnas.0703678104

4. Alvar J, Canavate C, Molina R, Moreno J, Nieto J. Canine leishmaniasis. Adv Parasitol (2004) 57:1-88. doi:10.1016/S0065-308X(04)57001-X

5. Nasereddin A, Baneth G, Schonian G, Kanaan M, Jaffe CL. Molecular fingerprinting of Leishmania infantum strains following an outbreak of visceral leishmaniasis in central Israel. J Clin Microbiol (2005) 43(12):6054-9. doi:10.1128/JCM.43.12.6054-6059.2005
6. Bader KA, Schnur LF, Nasereddin A, Pratlong F, Dedet JP, Shaheen L, et al. Palestinian infantile visceral leishmaniasis caused by a genetic variant of Leishmania infantum belonging to a new zymodeme. Trop Med Int Health (2005) 10(6):618-20. doi:10.1111/j.1365-3156.2005.01419.x

7. Desjeux P. Leishmaniasis. Public health aspects and control. Clin Dermatol (1996) 14(5):417-23. doi:10.1016/0738-081X(96)00057-0

8. Tiwary P, Kumar D, Singh RP, Rai M, Sundar S. Prevalence of sand flies and Leishmania donovani infection in a natural population of female Phlebotomus argentipes in Bihar State, India. Vector Borne Zoonotic Dis (2012) 12(6):467-72. doi:10.1089/vbz.2011.0808

9. Tiwary P, Kumar D, Mishra M, Singh RP, Rai M, Sundar S. Seasonal variation in the prevalence of sand flies infected with Leishmania donovani. PLoS One (2013) 8(4):e61370. doi:10.1371/journal.pone.0061370

10. Elnaiem DE. Ecology and control of the sand fly vectors of Leishmania donovani in East Africa, with special emphasis on Phlebotomus orientalis. J Vector Ecol (2011) 36(Suppl 1):S23-31. doi:10.1111/j.1948-7134.2011.00109.x

11. Dereure J, Rageh HA, Daoud W, Rioux JA. Eco-epidemiology of visceral and cutaneous leishmaniasis in the Arab Republic of Yemen. II. A survey using intradermal reaction to leishmanin in a zone of mixed infestation with Leishmania tropica, L. donovani and L. infantum. Bull Soc Pathol Exot Filiales (1989) 82(5):665-8.

12. Ready PD. Leishmaniasis emergence in Europe. Euro Surveill (2010) 15(10):19505.

13. Giorgobiani E, Lawyer PG, Babuadze G, Dolidze N, Jochim RC, Tskhvaradze L, et al. Incrimination of Phlebotomus kandelakii and Phlebotomus balcanicus as vectors of Leishmania infantum in Tbilisi, Georgia. PLoS Negl Trop Dis (2012) 6(4):e1609. doi:10.1371/journal.pntd.0001609

14. Silva ES, Gontijo CM, Pacheco RS, Fiuza VO, Brazil RP. Visceral leishmaniasis in the Metropolitan Region of Belo Horizonte, State of Minas Gerais, Brazil. Mem Inst Oswaldo Cruz (2001) 96(3):285-91. doi:10.1590/S007402762001000300002

15. Lainson R, Rangel EF. Lutzomyia longipalpis and the eco-epidemiology of American visceral leishmaniasis, with particular reference to Brazil: a review. Mem Inst Oswaldo Cruz (2005) 100(8):811-27. doi:10.1590/S0074-02762005000800001

16. Dorlo TP, Balasegaram M, Beijnen JH, de Vries PJ. Miltefosine: a review of its pharmacology and therapeutic efficacy in the treatment of leishmaniasis. J Antimicrob Chemother (2012) 67(11):2576-97. doi:10.1093/jac/dks275

17. Sundar S, Chakravarty J. Liposomal amphotericin B and leishmaniasis: dose and response. J Glob Infect Dis (2010) 2(2):159-66. doi:10.4103/0974-777X.62886

18. Chakravarty J, Sundar S. Drug resistance in leishmaniasis. J Glob Infect Dis (2010) 2(2):167-76. doi:10.4103/0974-777X.62887

19. Meheus F, Balasegaram M, Olliaro P, Sundar S, Rijal S, Faiz MA, et al. Costeffectiveness analysis of combination therapies for visceral leishmaniasis in the Indian subcontinent. PLoS Negl Trop Dis (2010) 4(9):e818. doi:10.1371/journal. pntd.0000818

20. Bern C, Haque R, Chowdhury R, Ali M, Kurkjian KM, Vaz L, et al. The epidemiology of visceral leishmaniasis and asymptomatic leishmanial infection in a highly endemic Bangladeshi village. Am J Trop Med Hyg (2007) 76(5):909-14.

21. Ribeiro JM, Francischetti IM. Role of arthropod saliva in blood feeding: sialome and post-sialome perspectives. Annu Rev Entomol (2003) 48:73-88. doi:10.1146/annurev.ento.48.060402.102812

22. Kamhawi S. The biological and immunomodulatory properties of sand fly saliva and its role in the establishment of Leishmania infections. Microbes Infect (2000) 2(14):1765-73. doi:10.1016/S1286-4579(00)01331-9

23. Andrade BB, de Oliveira CI, Brodskyn CI, Barral A, Barral-Netto M. Role of sand fly saliva in human and experimental leishmaniasis: current insights. Scand J Immunol (2007) 66(2-3):122-7. doi:10.1111/j.1365-3083.2007.01964.x

24. Oliveira F, de Carvalho AM, de Oliveira CI. Sand-fly saliva - man: the trigger trio. Front Immunol (2013) 4:375. doi:10.3389/fimmu.2013.00375

25. Belkaid Y, Kamhawi S, Modi G, Valenzuela J, Noben-Trauth N, Rowton E, et al. Development of a natural model of cutaneous leishmaniasis: powerful effects of vector saliva and saliva preexposure on the long-term outcome of Leishmania major infection in the mouse ear dermis. J Exp Med (1998) 188(10):1941-53. doi:10.1084/jem.188.10.1941

26. Carregaro V, Costa DL, Brodskyn C, Barral AM, Barral-Netto M, Cunha FQ, et al. Dual effect of Lutzomyia longipalpis saliva on Leishmania braziliensis infection is mediated by distinct saliva-induced cellular recruitment into BALB/c mice ear. BMC Microbiol (2013) 13:102. doi:10.1186/1471-2180-13-102 
27. Collin N, Gomes R, Teixeira C, Cheng L, Laughinghouse A, Ward JM, et al. Sand fly salivary proteins induce strong cellular immunity in a natural reservoir of visceral leishmaniasis with adverse consequences for Leishmania. PLoS Pathog (2009) 5(5):e1000441. doi:10.1371/journal.ppat.1000441

28. Gomes R, Oliveira F, Teixeira C, Meneses C, Gilmore DC, Elnaiem DE, et al. Immunity to sand fly salivary protein LJM11 modulates host response to vector-transmitted Leishmania conferring ulcer-free protection. J Invest Dermatol (2012) 132(12):2735-43. doi:10.1038/jid.2012.205

29. Gomes R, Teixeira C, Teixeira MJ, Oliveira F, Menezes MJ, Silva C, et al. Immunity to a salivary protein of a sand fly vector protects against the fatal outcome of visceral leishmaniasis in a hamster model. Proc Natl Acad Sci U S A (2008) 105(22):7845-50. doi:10.1073/pnas.0712153105

30. Kamhawi S, Belkaid Y, Modi G, Rowton E, Sacks D. Protection against cutaneous leishmaniasis resulting from bites of uninfected sand flies. Science (2000) 290(5495):1351-4. doi:10.1126/science.290.5495.1351

31. Oliveira F, Lawyer PG, Kamhawi S, Valenzuela JG. Immunity to distinct sand fly salivary proteins primes the anti-Leishmania immune response towards protection or exacerbation of disease. PLoS Negl Trop Dis (2008) 2(4):e226. doi:10.1371/journal.pntd.0000226

32. Tavares NM, Silva RA, Costa DJ, Pitombo MA, Fukutani KF, Miranda JC, et al. Lutzomyia longipalpis saliva or salivary protein LJM19 protects against Leishmania braziliensis and the saliva of its vector, Lutzomyia intermedia. PLoS Negl Trop Dis (2011) 5(5):e1169. doi:10.1371/journal.pntd.0001169

33. Valenzuela JG, Belkaid Y, Garfield MK, Mendez S, Kamhawi S, Rowton ED, et al. Toward a defined anti-Leishmania vaccine targeting vector antigens: characterization of a protective salivary protein. J Exp Med (2001) 194(3):331-42. doi:10.1084/jem.194.3.331

34. Morris RV, Shoemaker CB, David JR, Lanzaro GC, Titus RG. Sandfly maxadilan exacerbates infection with Leishmania major and vaccinating against it protects against L. major infection. J Immunol (2001) 167(9):5226-30. doi:10.4049/ jimmunol.167.9.5226

35. Teixeira C, Gomes R, Oliveira F, Meneses C, Gilmore DC, Elnaiem DE, et al. Characterization of the early inflammatory infiltrate at the feeding site of infected sand flies in mice protected from vector-transmitted Leishmania major by exposure to uninfected bites. PLoS Negl Trop Dis (2014) 8(4):e2781. doi:10.1371/journal.pntd.0002781

36. Peters NC, Egen JG, Secundino N, Debrabant A, Kimblin N, Kamhawi S, et al. In vivo imaging reveals an essential role for neutrophils in leishmaniasis transmitted by sand flies. Science (2008) 321(5891):970-4. doi:10.1126/science. 1159194

37. Peters NC, Kimblin N, Secundino N, Kamhawi S, Lawyer P, Sacks DL. Vector transmission of Leishmania abrogates vaccine-induced protective immunity. PLoS Pathog (2009) 5(6):e1000484. doi:10.1371/journal.ppat.1000484

38. Zahedifard F, Gholami E, Taheri T, Taslimi Y, Doustdari F, Seyed N, et al. Enhanced protective efficacy of nonpathogenic recombinant Leishmania tarentolae expressing cysteine proteinases combined with a sand fly salivary antigen. PLoS Negl Trop Dis (2014) 8(3):e2751. doi:10.1371/journal.pntd.0002751

39. Gomes RB, Brodskyn C, de Oliveira CI, Costa J, Miranda JC, Caldas A, et al. Seroconversion against Lutzomyia longipalpis saliva concurrent with the development of anti-Leishmania chagasi delayed-type hypersensitivity. J Infect Dis (2002) 186(10):1530-4. doi:10.1086/344733

40. Vinhas V, Andrade BB, Paes F, Bomura A, Clarencio J, Miranda JC, et al. Human anti-saliva immune response following experimental exposure to the visceral leishmaniasis vector, Lutzomyia longipalpis. Eur J Immunol (2007) 37(11):3111-21. doi:10.1002/eji.200737431

41. Oliveira F, Traore B, Gomes R, Faye O, Gilmore DC, Keita S, et al. Delayedtype hypersensitivity to sand fly saliva in humans from a leishmaniasis-endemic area of Mali is Th1-mediated and persists to midlife. J Invest Dermatol (2013) 133(2):452-9. doi:10.1038/jid.2012.315

42. Rosypal AC, Troy GC, Zajac AM, Frank G, Lindsay DS. Transplacental transmission of a North American isolate of Leishmania infantum in an experimentally infected beagle. J Parasitol (2005) 91(4):970-2. doi:10.1645/GE-483R.1

43. Kimblin N, Peters N, Debrabant A, Secundino N, Egen J, Lawyer P, et al. Quantification of the infectious dose of Leishmania major transmitted to the skin by single sand flies. Proc Natl Acad Sci U S A (2008) 105(29):10125-30. doi:10.1073/pnas.0802331105
44. Secundino NF, de Freitas VC, Monteiro CC, Pires AC, David BA, Pimenta PF. The transmission of Leishmania infantum chagasi by the bite of the Lutzomyia longipalpis to two different vertebrates. Parasit Vectors (2012) 5:20. doi:10.1186/1756-3305-5-20

45. Maia C, Seblova V, Sadlova J, Votypka J, Volf P. Experimental transmission of Leishmania infantum by two major vectors: a comparison between a viscerotropic and a dermotropic strain. PLoS Negl Trop Dis (2011) 5(6):e1181. doi:10.1371/journal.pntd.0001181

46. Oliveira F, Kamhawi S, Seitz AE, Pham VM, Guigal PM, Fischer L, et al. From transcriptome to immunome: identification of DTH inducing proteins from a Phlebotomus ariasi salivary gland cDNA library. Vaccine (2006) 24(3):374-90. doi:10.1016/j.vaccine.2005.07.085

47. Xu X, Oliveira F, Chang BW, Collin N, Gomes R, Teixeira C, et al. Structure and function of a "yellow" protein from saliva of the sand fly Lutzomyia longipalpis that confers protective immunity against Leishmania major infection. J Biol Chem (2011) 286(37):32383-93. doi:10.1074/jbc.M111.268904

48. Ready PD. Epidemiology of visceral leishmaniasis. Clin Epidemiol (2014) 6:147-54. doi:10.2147/CLEP.S44267

49. WHO. Control of the leishmaniases. Report of a WHO expert committee. World Health Organ Tech Rep Ser (2010) 949:1-200.

50. Vlkova M, Sima M, Rohousova I, Kostalova T, Sumova P, Volfova V, et al. Comparative analysis of salivary gland transcriptomes of Phlebotomus orientalis sand flies from endemic and non-endemic foci of visceral leishmaniasis. PLoS Negl Trop Dis (2014) 8(2):e2709. doi:10.1371/journal.pntd. 0002709

51. Valenzuela JG, Garfield M, Rowton ED, Pham VM. Identification of the most abundant secreted proteins from the salivary glands of the sand fly Lutzomyia longipalpis, vector of Leishmania chagasi. J Exp Biol (2004) 207(Pt 21):3717-29. doi:10.1242/jeb.01185

52. Anderson JM, Oliveira F, Kamhawi S, Mans BJ, Reynoso D, Seitz AE, et al. Comparative salivary gland transcriptomics of sandfly vectors of visceral leishmaniasis. BMC Genomics (2006) 7:52. doi:10.1186/1471-2164-7-52

53. Rohousova I, Subrahmanyam S, Volfova V, Mu J, Volf P, Valenzuela JG, et al. Salivary gland transcriptomes and proteomes of Phlebotomus tobbi and Phlebotomus sergenti, vectors of leishmaniasis. PLoS Negl Trop Dis (2012) 6(5):e1660. doi:10.1371/journal.pntd.0001660

54. Teixeira C, Gomes R, Collin N, Reynoso D, Jochim R, Oliveira F, et al. Discovery of markers of exposure specific to bites of Lutzomyia longipalpis, the vector of Leishmania infantum chagasi in Latin America. PLoS Negl Trop Dis (2010) 4(3):e638. doi:10.1371/journal.pntd.0000638

55. Aslan H, Dey R, Meneses C, Castrovinci P, Jeronimo SM, Oliva G, et al. A new model of progressive visceral leishmaniasis in hamsters by natural transmission via bites of vector sand flies. J Infect Dis (2013) 207(8):1328-38 doi:10.1093/infdis/jis932

56. Das S, Freier A, Boussoffara T, Das S, Oswald D, Losch FO, et al. Modular multiantigen $\mathrm{T}$ cell epitope-enriched DNA vaccine against human leishmaniasis. Sci Transl Med (2014) 6(234):234ra56. doi:10.1126/scitranslmed.3008222

57. Singh B, Sundar S. Leishmaniasis: vaccine candidates and perspectives. Vaccine (2012) 30(26):3834-42. doi:10.1016/j.vaccine.2012.03.068

58. Kamhawi S, Oliveira F, Valenzuela JG. Using humans to make a human leishmaniasis vaccine. Sci Transl Med (2014) 6(234):234fs18. doi:10.1126/scitranslmed. 3009118

59. Courtenay O, Carson C, Calvo-Bado L, Garcez LM, Quinnell RJ. Heterogeneities in Leishmania infantum infection: using skin parasite burdens to identify highly infectious dogs. PLoS Negl Trop Dis (2014) 8(1):e2583. doi:10.1371/journal. pntd.0002583

60. Reis AB, Giunchetti RC, Carrillo E, Martins-Filho OA, Moreno J. Immunity to Leishmania and the rational search for vaccines against canine leishmaniasis. Trends Parasitol (2010) 26(7):341-9. doi:10.1016/j.pt.2010.04.005

61. Ponce C, Ponce E, Morrison A, Cruz A, Kreutzer R, McMahon-Pratt D, et al. Leishmania donovani chagasi: new clinical variant of cutaneous leishmaniasis in Honduras. Lancet (1991) 337(8733):67-70. doi:10.1016/0140-6736(91) 90734-7

62. Pratlong F, Bastien P, Perello R, Lami P, Dedet JP. Human cutaneous leishmaniasis caused by Leishmania donovani sensu stricto in Yemen. Trans $R$ Soc Trop Med Hyg (1995) 89(4):398-9. doi:10.1016/0035-9203(95)90025-X 
63. Marty P, Pratlong F, Marcelet B, Adda A, Le Fichoux Y. Leishmania infantum variant MON-24 isolated from a cutaneous lesion acquired in the suburban area of Nice (France). Parasite (1994) 1(2):175-6.

64. Svobodova M, Alten B, Zidkova L, Dvorak V, Hlavackova J, Myskova J et al. Cutaneous leishmaniasis caused by Leishmania infantum transmitted by Phlebotomus tobbi. Int J Parasitol (2009) 39(2):251-6. doi:10.1016/j.ijpara.2008. 06.016

65. Siriwardana HV, Noyes HA, Beeching NJ, Chance ML, Karunaweera ND, Bates PA. Leishmania donovani and cutaneous leishmaniasis, Sri Lanka. Emerg Infect Dis (2007) 13(3):476-8. doi:10.3201/eid1303.060242

66. Karunaweera ND. Leishmania donovani causing cutaneous leishmaniasis in Sri Lanka: a wolf in sheep's clothing? Trends Parasitol (2009) 25(10):458-63. doi:10.1016/j.pt.2009.07.002

67. Gradoni L, Gramiccia M. Leishmania infantum tropism: strain genotype or host immune status? Parasitol Today (1994) 10(7):264-7. doi:10.1016/0169-4758(94) 90142-2
Conflict of Interest Statement: The authors declare that the research was conducted in the absence of any commercial or financial relationships that could be construed as a potential conflict of interest.

Received: 30 May 2014; accepted: 14 July 2014; published online: 08 August 2014. Citation: Kamhawi S, Aslan H and Valenzuela JG (2014) Vector saliva in vaccines for visceral leishmaniasis: a brief encounter of high consequence? Front. Public Health 2:99. doi: 10.3389/fpubh.2014.00099

This article was submitted to Immunotherapies and Vaccines, a section of the journal Frontiers in Public Health.

Copyright (c) 2014 Kamhawi, Aslan and Valenzuela. This is an open-access article distributed under the terms of the Creative Commons Attribution License (CC BY). The use, distribution or reproduction in other forums is permitted, provided the original author(s) or licensor are credited and that the original publication in this journal is cited, in accordance with accepted academic practice. No use, distribution or reproduction is permitted which does not comply with these terms. 